\title{
Agility and Human factors in the Virtual Enterprise
}

\author{
Florent L. M. FREDERIX \\ ALCATEL - MICROELECTRONICS \\ Westerring 15, 9700 Oudenaarde, Belgium, \\ tel. +32 (055) 332231, fax. +32 (055) 332452, \\ email: florent.frederix@mie.alcatel.be
}

\begin{abstract}
This thematic paper based on experiences obtained with X-CITTIC (1995) in semiconductor supply chains introduces the concepts "Agility" and "Human Sensor" in "Virtual Enterprises" found in the announced HUTOP (1998) project.

Virtual enterprises are a new industrial paradigm as more and more companies reduce their wholly owned ability to a core competence and run lean. They employ outsourced service providers for a large range of operations including manufacture and transportation provisions. The Agility, or the continuous change, of virtual enterprises, production processes and production equipment's creates a real issue for currently existing Supply Chain solutions. The application can be outdated even before it reaches the maturity needed for an error free deployment.

This article identifies area's in which the Human sensor - knowledge acquisition, probably the most important and in the past often neglected, factor of the whole enterprise, can be used to improve "Virtual Enterprise" management.
\end{abstract}

Keywords

Agility, Human Sensor, Virtual Enterprise, Knowledge Aquisition, Planning, Neural Nets, Proxy agents

\section{AGILITY IN GLOBAL MANUFACTURING}

Agility of the whole global manufacturing and distribution chain is an important reason to integrate the human factor in the design of supply chain applications. The 
next paragraphs show the influence of "Agility" and the "Human sensor" at the supply chain and enterprise entity level for the complete manufacturing cycle.

\subsection{Agility at the level of the virtual enterprise}

The pace of advancement in industry and production techniques has probably never been faster. New products and markets emerge with unprecedented speed. Production chains consist of units located all over the world (see paragraph on "economic drivers for agility").

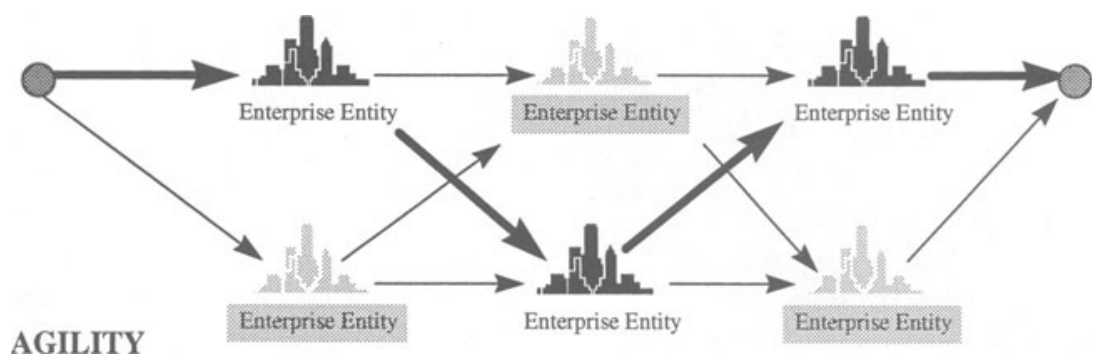

* Subcontractors or partners are order and time dependent,

* Some subcontractors can replace one or more enterprise entities.

\section{HUMAN FACTOR}

* Assist in complex decision processes (e.g. Partners and intermediate stock levels),

* Highly flexible and performant algorithms required,

* Design products for supply chain constructs. (e.g. suspend product configurability to last steps)

\section{Figure 1 : Illustration of Virtual Enterprise Constructs}

The concept of a virtual enterprise consisting of owned individual units and independent subcontractors creates more agility and increases efficiency (Figure 1). The importance of this factor has already resulted in tremendous changes all over the industry ${ }^{1}$. Large conglomerates have been split in independent units and new global competing groups have been formed in a few years. ${ }^{2}$. The most striking example is probably that two industrial partners ${ }^{3}$ in the X-Cittic project have been separated from their original supply chain and are currently partner of another one. These extreme examples of changes in extended enterprises are not the most frequent changes which are observed in the virtual manufacturing and distribution enterprises to which they belong, but changes that are the result of market-, customer and material flow dynamics make it often necessary to speedily reconfigure a virtual enterprise and also to swiftly renegotiate with partners (including subcontractors) in the enterprise to manage any critical disruptions to plan in order to protect delivery precision. Currently the embryonic supply chain

\footnotetext{
${ }^{1}$ Large groups (IBM) split into units (Lexmark) that compete with other subcontractors (Canon).

${ }_{3}^{2}$ Cisco, a merger of companies is a fierce competitor against established gigants in the telecom market.

${ }^{3}$ TEMIC from the Daimler-Benz group and GEC-Plessey from the GEC Cooperation in 1998.
} 
systems and tools do not sufficiently deal with the partner collaboration within a virtual enterprise nor the interaction of people to prevent a high inertia at times to resolve order flow disruptions.

A similar trend has also been confirmed in completely other industries and described by Kohli (1994) in his article "Coordinating Buyer-Seller transactions Across Multiple Products".

\subsection{Agility at the enterprise entity / shop floor level}

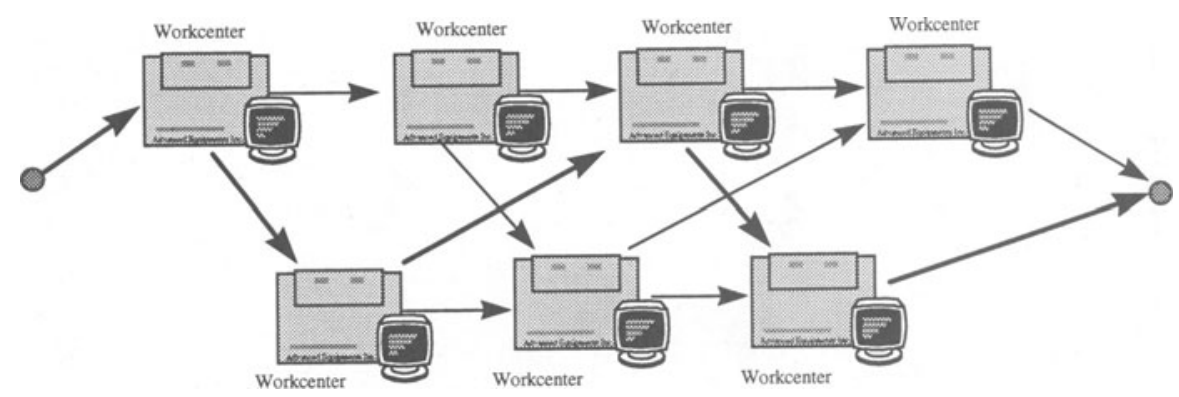

Route of products in the enterprise entity. Small lines present optional processing path's

\section{AGILITY}

* Processes and process routes are subject to learning

- process steps can be merged,

- process yield and throughput changes.

${ }^{*}$ New or improved products require other process routes

\section{HUMAN FACTOR}

* Design for manufacturability,

* Split process steps in flexible, human centered parts and parts suited for automation.

\section{Figure 2 : Illustration of agility at the enterprise entity / shop floor level}

Process routes and processes are subject to adjustments for new product or improved product introductions, production learning cycles and process yield and throughput changes. All these changes are taking place continuously and concurrently and determine to a large extent the efficiency of the processes at the local site (Figure 2).

The challenge to manage the explosion of variety in the so called 'single lot' processing has called for a new understanding of working methods, and new systems for planning and control. Improvements can be made through the product design (design for manufacturability) and in the flexible production chain organisation (easily reconfigurable production processes). 


\section{ECONOMIC FACTS THAT STIMULATE VIRTUAL SEMICONDUCTOR ENTERPRISE AGILITY.}

Facts that stimulate agility:

- Competitivity in the current global economic context means the ability to allocate and manage units at the best fitted location. Agile (virtual) multi site planning and control tools that take into account collaborating partnerships and globally distributed manufacturing and distribution, fast internet communications and real time planning and control are key to succeed.

- The high pace of innovation in semiconductor and this linked to investment levels that nearly reach the budget of a nuclear power plant requires very flexible and lean concepts that allow a high occupation of the installed equipment's $s^{4}$ and at the same time can adapt rapidly to changing technologies ${ }^{5}$.

- The pace of integrated circuit innovation is even higher, four times more performant products are introduced every year (this means that a product ages in less than a year).

- A large number of manufacturing companies which operate in Europe and the USA use the flexibility and customer responsiveness that the market asks as a competitive weapon against the "new economic world" that excels in low cost mass production.

- The creation of a virtual production chain that integrates all distribution and production units that participate in the final product provides large advantages ${ }^{6}$. (An integrated circuit is part of a complex telephone exchange system, computer, car, ... )

Due to these drivers it becomes more and more clear that the key to success is not only found in manufacturing performance but in the improvement of business capability. Business capability is about capacity, ability and the essential elements needed to meet customer demands and be responsive to changes. Capability can be defined as that what can be achieved in contrast with performance. Business capability also means the control that the core company has over the manufacturing, transport and distribution processes, from order placement to customer delivery and this across the whole virtual enterprise. These pressures to create flexible semiconductor virtual enterprises have not passed unnoticed by researchers and an interesting analysis of causes can be found in Monteverde (1995).

\footnotetext{
${ }^{4}$ A SEMATECH study has shown that resource utilization can increase with $30 \%$.

${ }^{5}$ Semiconductor sites build 10 years will not resemble today's plants (quick progress in engineering).

${ }^{6}$ ALCATEL : An internal survey revealed that a working supply chain model can reduce product lead times, delay capital binding and accelerate the introduction of new designs in production.
} 

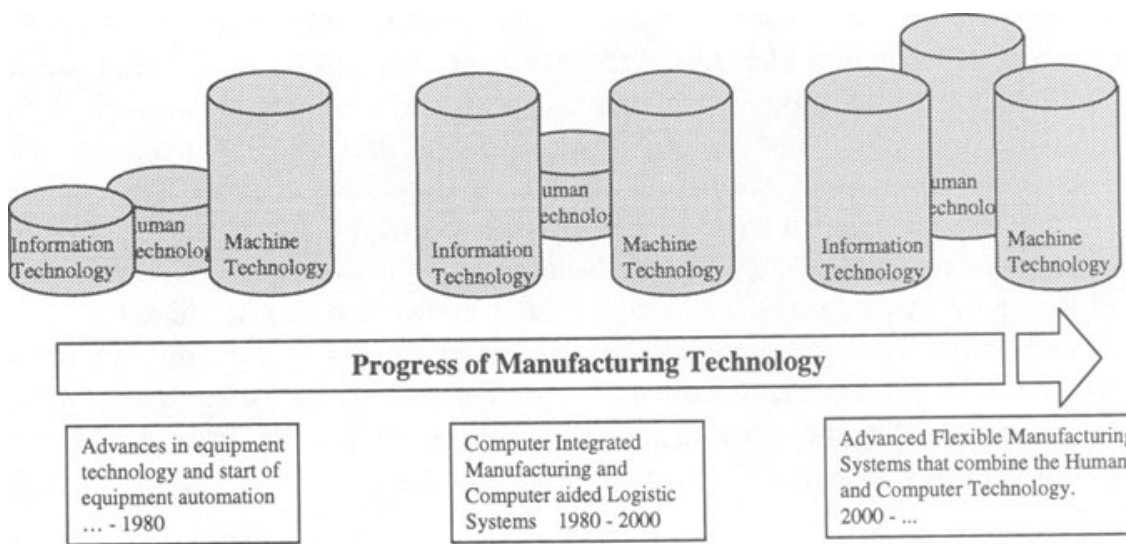

\section{Figure 3: The importance of the Human factor}

As shown in Figure 4 human factors have been largely neglected in the past. Progress has been primarily the result of advances in machine technology in the eighties and information technology (Computer Integrated Manufacturing systems) in the nineties.

This paper has already introduced "agility" and "progress" as major difficulties for the currently existing virtual enterprise planning and control applications, the remaining part of this text will be spend on the human knowledge creation process and how this can be used to design new and improved applications.

Ikujiro Nonaka and Hirotaka Takeuchi (1995) have recently written an interesting book - rewarded with the title of best book on management of 1995 - on knowledge creation. It gives a description how human learning processes work and a short summary of these learning principles will be given and the next paragraphs

\subsection{Dimensions of knowledge creation}

Two dimensions of knowledge creation exist:

- The ontological dimension starts from the knowledge that is created by an individual. This knowledge is "amplified" and "crystallized" by distributing this knowledge in the enterprise and expanding due to "community interaction". This community interaction can cross intra- and interorganizational levels and boundaries.

- The epistemological dimension is pointing at the distinction between tacit knowledge and explicit knowledge. Tacit knowledge is personal, context specific, and therefore hard to formalize and communicate. Explicit or "codified" knowledge, on the other hand, refers to knowledge that is 
transmittable in formal, systematic language. Polanyi (1966) contends that human beings acquire knowledge by actively creating and organizing their own experiences. This tacit knowledge includes cognitive and technical elements. Cognitive elements or mental models (Johnson-Laird, 1983) such as schemata, paradigms,... help individuals to perceive and define their world and at the other hand the technical elements of tacit knowledge includes concrete know-how, crafts and skills. It is important to note here that the cognitive elements of tacit knowledge refer to an individual's images of reality and visions for the future, that is "what is" and "what ought to be". It is a key factor for creating new knowledge. Tacit knowledge can be shared between individuals through communication and is an analog process that requires a kind of "simultaneous processing" of the complexities of issues shared by the individuals. On the other hand, explicit knowledge is about past events or objects and is oriented toward a context free theory.

\subsection{The modes of knowledge conversion and the knowledge spiral}

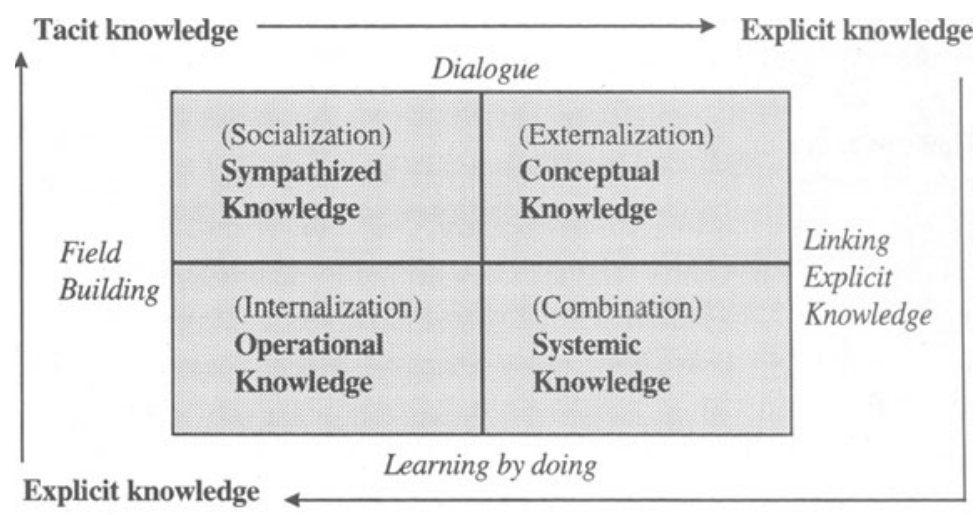

\section{The knowledge spiral}

\section{Figure 4 : The knowledge spiral}

The knowledge spiral (Figure 5) consists of the following four steps of knowledge conversion that are repeated and in this way increase the knowledge of the system:

1. Socialization is a process of sharing experience and thereby creating tacit knowledge such as shared mental models and technical skills. The key to acquiring tacit knowledge is experience.

2. Externalization is a process of articulating tacit knowledge into explicit concepts. It is a quintessential knowledge-creation process in that tacit 
knowledge becomes explicit, taking the shapes of metaphors, analogies, concepts, hypotheses or models. Among the four modes of knowledge conversion, externalization holds the key to knowledge creation, because it creates new explicit concepts from tacit knowledge.

3. Combination is a process of systematizing concepts into a knowledge system. This mode of knowledge conversion involves combining different bodies of explicit knowledge.

4. Internalization is a process of embodying explicit knowledge into tacit knowledge. This occurs if combined knowledge is converted into real products and product designs. This process is closely related to "learning by doing" and will create a richer tacit knowledge.

\section{A VIRTUAL ENTERPRISE MANAGEMENT SYSTEM INTEGRATING HUMAN FACTOR CONCEPTS}

From the first paragraphs it became clear that the Supply Chain is "agile" and difficult to manage using traditional paradigms (fixed algorithms based on systemic knowledge). It is therefore necessary to develop systems which deal with more versatile and flexible capacity models employed in order taking, planning and virtual enterprise scheduling, and event driven reactive virtual enterprise schedule repair to improve the control of on time delivery precision of flow changes which have a low inertia for their control resolution.

The goal of the work announced in this paper is to indicate how the human tacit knowledge aquisition process will be emulated in an advanced virtual enterprise management application. The prototype of the new supply chain management application will consist of two functional modules:

- a Proactive Reasoner, able to predict future virtual enterprise behavior,

- and the on line Assisted Dynamic Negotiator that supports subcontracting activities. 


\subsection{The neural network technology}

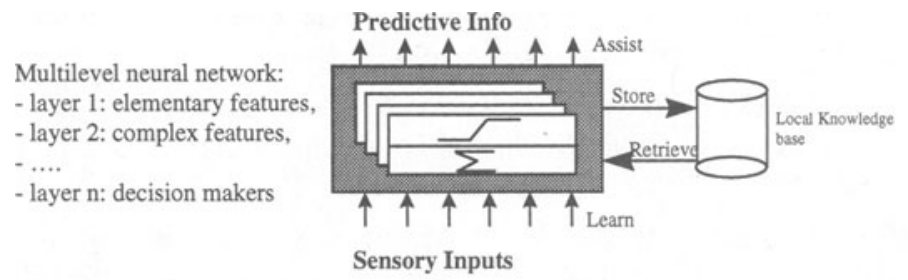

Task:

* Predictive Planning or

* On Line Negotiator

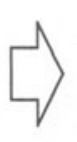

\section{Model}

* Process model,

* Translate in neural layers
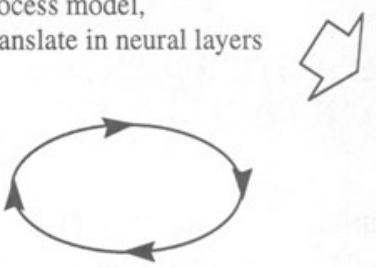

Key parameters

* Inputs,

* Outputs

Result: Prediction based

* on Sensory inputs,

* Knowledge base

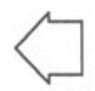

Problem Solving Activity

* Learning,

* Predicting

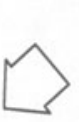

Figure 5 : Neural network technology to emulate tacit knowledge accumulation

To translate tacit knowledge into "computer behavior" neural network technology will be used. The complexity is in the definition of the different layers of the neural network based on the application model and the information available in the knowledge base.

Sensory inputs (Figure 6) of the network capture the behavior of the physical system and the outputs predict the comportment of the real system taken all available information in consideration.

Thomas Schmidt describes in a paper (Westkamper et al., 1997) at the Brazil conference how neural network technology can be used to model queue netwerk based planning systems. More general mathematical models of neural network based systems can be found in "Introduction to the theory of Neural Computation" (Hertz et al., 1989).

\subsection{The Proactive Reasoner}

The Proactive Reasoner is an interactive, distributed simulator, using artificial neural networks to incorporate human "knowledge" through a learning component, 
that will be able to predict how the supply chain behaves in the future. Traditional virtual enterprise planning applications that count on an explicit model consisting of registered resources, set-up and working times,... are difficult to maintain and can change slowly or unnoticed. Furthermore because some supply chain partners are subcontractors no complete information will be available.

Neural networks on the other hand emulate the human sensor "tacit learning" comportment because they can be trained for certain behaviors. In that way, the attributes of the resources - and thus their capacities - are implicitly included even if sensitive key data such as costs, yield, stock and utilization remain hidden (in the case of independent subcontractors). Especially protection of data and the ease of update do not exist when applying conventional methods such as pure discrete event simulation. Furthermore, neural nets make the standardized abstraction of resources for the entire virtual enterprise possible.

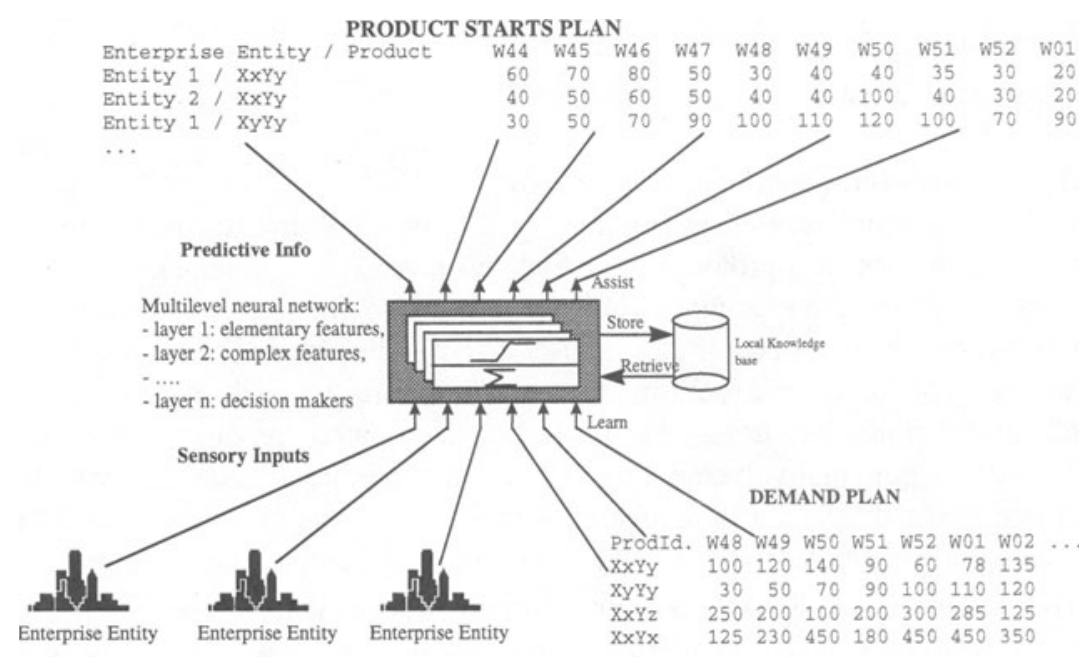

\section{Figure 6 : Neural network based Proactive Reasoner}

The goal of this "Proactive Reasoner" is to allow for a much better predictability and transparency of the manufacturing process across the virtual enterprise. By its multi-level approach to modeling it will be capable of serving more than one planning level in rapidly generating reliable prediction of future manufacturing states and what-if scenarios.

The key performance indicators to measure the efficiency of this "Proactive Reasoner" are not different from other supply chain planning applications: schedule work such that all customer orders are met on time with low inventory levels, short lead times and at the lowest production cost. Unfortunately these goals are conflicting. We can meet customer orders relying on large inventory 
levels and in a similar way, we can keep lead times short if we invest capital in a large number of machines and keep their utilization low. Thus the goal of this proactive planning and scheduling application is to find the best trade off between these objectives to ensure profitability of the virtual enterprise.

The challenge of virtual enterprise planning is to balance the requirements of reliable, fast customer delivery with manufacturing-, inventory-, delivery- and product obsolete costs. The virtual enterprise planning tools should also pinpoint the area's and production sites that need adapted capacity. A successful implementation of virtual planning can be measured by the ability of a member company to:

- do immediate order acceptance with commitment to due date, quantity and quality;

- adjust orders to changing customer requirements;

- more delivery accuracy;

- shorter production time (of the complete chain);

- lower buffer stocks.

\subsection{The Assisted Dynamic Negotiator}

This is a tool which formalises negotiations through goal-oriented proxy agents which follow a defined protocol and which have access to requisite knowledge and protected information making use of fast Internet/communication technology. Such proxy agents acting on behalf of organisations or people assigned to specific planning, scheduling, or expediting tasks will allow faster decision making in a global environment or virtual enterprises. It is anticipated that this negotiation tool will alleviate human involvement by reducing the number of tasks and will also enhance the quality of decision making where people have to be involved. JAVA ${ }^{7}$ is a candidate technology for the human interface of the dynamic negotiator.

The assisted dynamic negotiator works in an environment of inter company (and often - intercontinental) co-operation and this implies some social and security hurdles. Every partner in the chain can behave co-operative, self interested and hostile:

- Co-operative partners attempt to maximise social welfare, which is the sum of all partner's interest. They are willing to take individual losses in service of the good of the society of partners.

- The model found in multi-enterprise manufacturing consisting of individual companies is likely this of a self-interested agent - it wants to maximise its own profit while not caring about the other partner companies' profits within the virtual enterprise.

${ }^{7}$ JAVA : Trade mark of Sun Microsystems, object oriented language for internet applications. 
- A third type of relationship that occurs in distributed manufacturing is hostile. As an example, one can consider partner companies that compete against each other in the same market. In such a setting the partner can be viewed as maximising their utility which increases with their own gains but also with other partners' losses. Even if a partner is self-interested on a strategic level, it may be of its interest to act in a hostile manner at the operative level.

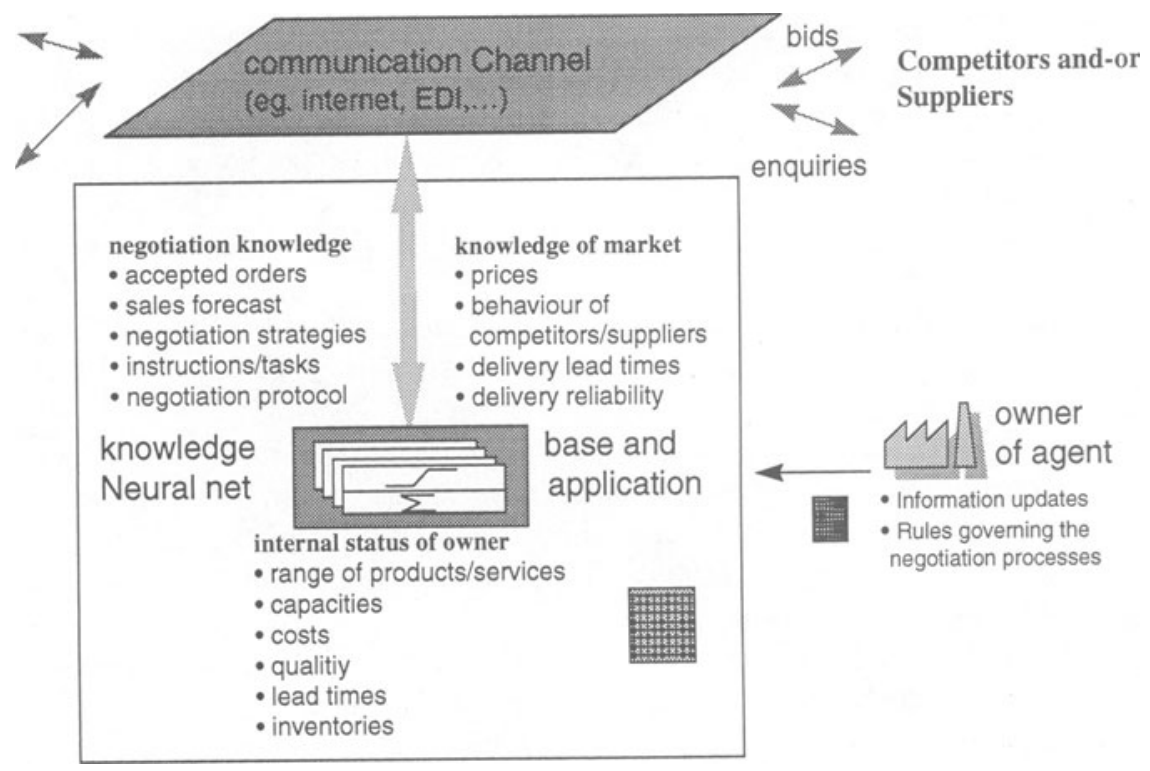

\section{Figure 7: The Assisted Dynamic Negotiator (Rupp 1998)}

In many situations, the distributed virtual enterprise actually comprises partners of more than one of the classes (Neiman et al., 1994). The assisted dynamic negotiator application will have to implement the security measures and the functionality required to avoid all the issues that can result from the partner behaviour specified in the previous paragraph.

\section{CONCLUSIONS}

This paper outlines the need for advanced agile virtual enterprise planning and negotiation tools. It also has shown that technology and economic drivers are in place to stimulate this project.

The complexity of the problem, the issue of integration and the user context of the agile virtual enterprise application that spans different corporate cultures remain the major hurdles to be taken. However in semiconductor- and other industries is the availability of this type of application identified as very important and critical for future business perspectives (Gilles et al., 1995). 


\section{REFERENCES}

Gilles, J. and Puttick J. (1995) Factory of the future, CEC Eureka Project, Factory EI 1003 - Final Report Synopsis.

Hertz J., Krogh A., Palme R.G. (1989), Introduction to the theory of Neural Computation, Lectue Notes Volume 1, Santa Fe Institute - Studies in the Sciences of Complexity, Addison-Wesley Publishing Company, Reading, Massachusetts.

HUTOP (1999 - 2002) Human Sensory Factors for Total Product Life Cycle, IMS project proposal.

Kohli R., Park H. (1994) Coordinating Buyer-Seller Transactions Across Multiple Products, Management Science, Vol. 40, No 9, September 1994, pg. 1145 1150, Maryland.

Johnson-Laird, P.N. (1983), "Mental Models". Cambridge University Press, Cambridge

Monteverde K. , (1995) Technical Dialog as an Incentive for Vertical Integration in the Semiconductor Industry, Management Science, Vol 41, No. 10, October 1995 , Maryland.

Neiman D., Hildum D., Lesser V.R., and Sandholm T.W. (1994) Exploiting metalevel information in a distributed scheduling system. Proceedings Twelfth National Conference on Artificial Intelligence (A A A I 94), Aug. 1994.

Nonaka I., Takeuchi H. (1995) The knowledge Creating Company, Oxford University Press, Oxford, USA.

Polanyi M. (1966) The Tacit Dimension. Routledge and Kegan Paul, London.

Rupp T. (1998) HUTOP working paper, Imperial College.

Westkamper E., Schmidt T. (1997) Concept of a Learning Simulation System, MCPL'97 IFAC/IFIP Conference on Management and Control of Production and Logistics - Conference Proceedings. Campinas, SP, Brazil.

X-CITTIC (1996-1998) A Planning and Control System for Semiconductor Virtual Enterprises, Esprit project no. EP20544.

\section{BIOGRAPHY}

The author holds an Electrical Engineering (1978) and Computer Science (1982) degree, obtained a Master of Business Administration in 1986 and is currently also active as Postgraduate Researcher in Applied Economics.

In his professional career, he has been software development manager for 9 years, during which his team succeeded to design one of world's best line-art design system's (Seybold Report on Publishing systems of 1989) and for the last 7 years he holds a senior management position at Alcatel. Currently he is also European Steering Board member of the world-wide "HUTOP" project. 